\title{
The development and validation of the King's Brief Interstitial Lung Disease (K-BILD) health status questionnaire
}

\author{
Amit S Patel, ${ }^{1}$ Richard J Siegert, ${ }^{2}$ Katherine Brignall, ${ }^{1}$ Patrick Gordon, ${ }^{3}$ Sophia Steer, ${ }^{3}$ \\ Sujal R Desai, ${ }^{4}$ Toby M Maher, ${ }^{5}$ Elisabetta A Renzoni, ${ }^{5}$ Athol U Wells, \\ Irene J Higginson, ${ }^{2}$ Surinder S Birring ${ }^{1}$
}

${ }^{1}$ Division of Asthma, Allergy and Lung Biology, King's College London, London, UK

${ }^{2}$ Department of Palliative Care, Policy and Rehabilitation, King's College London, Cicely Saunders Institute, London, UK

${ }^{3}$ Department of Rheumatology, King's College Hospital, London, UK

${ }^{4}$ Department of Radiology, King's College Hospital, London, UK

Interstitial Lung Disease Unit, Royal Brompton Hospital, London, UK

\section{Correspondence to}

Dr Surinder S Birring, Division of Asthma, Allergy and Lung Biology, King's College London, Denmark Hill, London SE5 9RS, UK; surinder.birring@nhs.net

Received 2 January 2012 Accepted 21 March 2012 Published Online First 3 May 2012

\section{ABSTRACT}

Rationale Health status is impaired in patients with interstitial lung disease (ILD). There is a paucity of tools that assess health status in ILD. The objective of this study was to develop and validate the King's Brief Interstitial Lung Disease questionnaire (K-BILD), a new health status measure for patients with ILD.

Methods Patients with ILD were recruited from outpatient clinics. The development of the questionnaire consisted of three phases: item generation; item reduction, allocation to domains by factor analysis, Rasch analysis to create unidimensional scales and validation; and repeatability testing.

Results 173 patients with ILD (49 with idiopathic pulmonary fibrosis) completed a preliminary 71-item questionnaire. 56 items were removed due to redundancy, low factor loadings or poor fit to the Rasch model. The final version of the K-BILD questionnaire consisted of 15 items and three domains (breathlessness and activities, chest symptoms and psychological). Internal consistency assessed with Cronbach's $\alpha$ coefficient was 0.94 for the K-BILD total score. Concurrent validity of the K-BILD questionnaire was high compared with St George's Respiratory Questionnaire $(r=0.90)$ and moderate with lung function (vital capacity, $r=0.50)$. The K-BILD questionnaire was repeatable over 2 weeks $(n=44)$, with intraclass correlation coefficients for domains and total score 0.86-0.94. The K-BILD construct validity for patients with idiopathic pulmonary fibrosis was similar to that of other ILDs.

Conclusion The K-BILD questionnaire is a brief, valid, self-completed health status measure for ILD. It could be used in the clinic to assess ILD from the patients' perspective.

\section{INTRODUCTION}

Interstitial lung diseases (ILDs) are characterised by inflammation and fibrosis of the lung parenchyma and they have an estimated incidence of 26-32 per $10^{5}$ persons per year. ${ }^{1}$ There is significant morbidity and mortality associated with ILD. The health status of patients with ILD is often impaired due to symptoms such as dyspnoea that limit physical activity. $^{2}$ An important goal of therapies for ILD should be to improve health status. Health status can be measured with disease-specific instruments, which compared with generic instruments are more

\section{Key messages}

What is the key question?

- To develop a brief tool to assess the health status of patients with interstitial lung disease.

What is the bottom line?

- King's Brief Interstitial Lung Disease questionnaire is a validated tool suitable for clinical and research use.

Why read on?

- The King's Brief Interstitial Lung Disease questionnaire may become an important outcome parameter in the evaluation of therapies for interstitial lung disease.

focused and responsive. There is a paucity of easy to use, disease-specific health status instruments for ILD, particularly conditions other than idiopathic pulmonary fibrosis (IPF). We set out to develop and validate an ILD-specific health status questionnaire. Our aim was to develop a tool that was brief, easy to administer and well validated.

\section{METHODS}

\section{Phase 1: item generation}

A preliminary King's Brief Interstitial lung disease (K-BILD) questionnaire was developed following review of health status and ILD literature; review of available health status questionnaires; face-to-face semi-structured interviews with 10 patients with a range of ILDs; and a multidisciplinary team meeting, consisting of respiratory, palliative care, rheumatology, academic and general physicians, a nurse, pharmacist, social worker and physiotherapist. The in-depth patient interviews aimed to identify health status issues and explore the impact of their lung condition. The interviews were repeated until they no longer yielded new themes. The interviews began with open-ended questions, for example, "What is it like to live with your lung condition?' and 'How does your lung condition affect you?' Patients were also asked questions about issues anticipated to be relevant to them and to comment on a preliminary K-BILD questionnaire. The questionnaire was worded to assess 
health status during the past 2 weeks and patients responded on a seven-point Likert scale.

\section{Subjects}

The preliminary K-BILD questionnaire was evaluated in patients with ILD recruited prospectively from secondary care (King's College Hospital) and tertiary care (Royal Brompton Hospital) specialist clinics from January to December 2010. Clinical characteristics, comorbidities and medications were recorded using a structured questionnaire. Patients selfcompleted health status questionnaires independently when attending clinic. The questionnaires were administered by a member of the research team not involved in the clinical care of the patient. Patients were excluded from the study if they could not read the questionnaire or completed less than $85 \%$ of the questionnaire. The cause of the ILD was determined by a multidisciplinary meeting of clinicians, radiologists and pathologists, following review of clinical characteristics, highresolution CT scan, lung function, and lung biopsy if available. The classification of ILD was consistent with international guidelines. ${ }^{3}{ }^{4}$ Patients were asked 'did you find the questionnaire difficult to complete?'

\section{Phase 2: item reduction, allocation of items to domains, Rasch analysis and validation of the questionnaire Item reduction}

Items demonstrating a minimum (floor effect) or maximum response (ceiling effect) of $\geq 60 \%$ were removed. ${ }^{5}$ The weaker items of highly inter-correlated item pairs $(r>0.8)$ as determined by face validity were also removed.

\section{Factor analysis}

The remaining items were subjected to exploratory factor analysis with varimax rotation and a scree plot to identify groups of related items or factors. ${ }^{6}$ Items that loaded high $(>0.5)$ on a single factor were retained. ${ }^{7}$

\section{Rasch analysis}

The Rasch model provides a template for testing the validity of a questionnaire as a unidimensional measure of the construct it was designed to assess. ${ }^{8}{ }^{9}$ It assumes that the response to an item is determined by two factors; the health status of the person and health status impairment represented by the item. A questionnaire that meets the requirements of a Rasch model has interval scaling properties. Patients are more likely to respond to items assessing less severe health status (difficulty) and less likely to items assessing more severe health status. Rasch analysis is an iterative process whereby the poorest fitting item is removed and the remaining items retested until a reliable, unidimensional scale is identified.

Rasch analysis was applied to the K-BILD domains and then all items combined to create an overall scale. The item-trait interaction assessed the questionnaire fit to a unidimensional model. A significant $\chi^{2}$ test for the overall item-trait interaction or individual items indicated misfit to the model and items were therefore removed. Item fit residuals, a summation of the difference between the observed score and that expected by the model for all persons, $\leq-2.5$ or $\geq 2.5$ indicated the items did not measure the same attribute as the domain and were also removed. ${ }^{10}$ Summary item and person fit residuals were item-person interaction statistics transformed to approximate a z score; a mean (SD) residual of approximately 0 (1) indicated good fit to the model. The Person Separation Index was determined to assess the ability of the K-BILD questionnaire to discriminate patients with different levels of health status impairment and test the reliability of the fit statistics. Internal consistency was assessed using Cronbach's $\alpha$ coefficient. The Likert response scale weightings for individual items were combined if necessary to ensure they detected progressive changes in health status. ${ }^{11}$ The K-BILD domain and total scores were transformed to a range of 0-100 ((actual score-lowest possible score/range) $\times 100$ ); $100=$ best health status. The influence of age, gender, ethnicity, immunosuppressant medication, presence of connective tissue disease (CTD vs no CTD) and ILD diagnosis on the patient's response to items was assessed for differential item functioning (DIF). The final version of the KBILD questionnaire was retested with Rasch analysis and confirmatory factor analysis.

\section{Concurrent validity}

Concurrent validity, which is the assessment of an instrument against other standards that provide an indication of the true value for the measurements, was assessed by investigating the relationship between K-BILD, lung function and other health status questionnaires. Forced vital capacity (FVC) and transfer factor of the lungs for carbon monoxide (TLCO) were assessed according to American Thoracic Society standards within 1 month of completion of questionnaires. ${ }^{12}{ }^{13}$ Patients completed the St George's Respiratory Questionnaire (SGRQ) and the Short Form-36 (SF36) questionnaire, which assessed respiratory and general health status respectively. ${ }^{14}{ }^{15}$ The sequence of administration of questionnaires was K-BILD, SF36 and SGRQ. The questionnaires were administered prior to the patient being assessed by the clinician or undergoing other procedures. The impact of the severity of ILD was assessed by investigating the health status of patients prescribed home oxygen compared with those not prescribed oxygen; patients using home oxygen were expected to have worse health status. The health status of patients with CTD-non-specific interstitial pneumonia (NSIP) and idiopathic NSIP was assessed to investigate the impact of CTD; CTD was not expected to affect ILDspecific health status. The independent predictors of health status were also determined.

\section{Phase 3: repeatability}

A subgroup of unselected consecutive patients with IPF and other ILDs completed the K-BILD questionnaire on two occasions, 2 weeks apart to investigate repeatability. Only patients whose condition was considered stable by both the patient and physician were recruited. Patients were excluded if they had a recent respiratory tract infection or change in medication within the previous month.

\section{Analysis}

SPSS software, V.18 and RUMM 2030 (RUMM Laboratory) were used for statistical analysis. Mean and SD was used to describe parametric data. $\mathrm{p}<0.05$ was considered significant. Correlations between parameters were assessed with Pearson's (r) coefficient. Univariate analysis was performed to identify associations between health status and age, gender, smoking, FVC\%, TLCO\%, medications and oxygen use. Multivariate analysis was performed to determine independent predictors of health status; significant variables identified from univariate analysis were entered as independent variables. The repeatability of the K-BILD questionnaire was assessed with intraclass correlation coefficients and a Bland Altman plot. The $95 \%$ limit of agreement was calculated as $1.96 \times \mathrm{SD}$ of within-subject differences. All patients gave written informed consent and the 
study was approved by the Research Ethics Committee (London-Surrey Borders).

\section{RESULTS}

\section{Phase 1: item generation and subject characteristics}

A preliminary version of the K-BILD questionnaire consisting of 71 items was developed following the item generation process. Ten patients with ILD (three IPF, three CTD-ILD, two idiopathic NSIP, two cryptogenic organising pneumonia) were interviewed to identify common themes and items. The interviews were discontinued when they did not yield new items. The preliminary K-BILD items covered a range of health topics that included breathlessness, other respiratory symptoms, activities, social impact, psychological health, medications, healthcare and relationships. The preliminary K-BILD questionnaire was evaluated in 10 patients with ILD to assess item wording and suitability; no further changes were made. The K-BILD questionnaire was administered to 173 patients with ILD to investigate validity (IPF (48 patients), CTD-ILD (67), idiopathic NSIP (20), hypersensitivity pneumonitis (16), cryptogenic organising pneumonia (8), pulmonary Langerhan's cell histiocytosis (4), lymphangioleiomyomatosis (3), respiratory bronchiolitis ILD (2), druginduced ILD (2), radiation fibrosis (1), lymphoid interstitial pneumonia (LIP) (1) and eosinophilic pneumonia (1); table 1). Sixty-seven patients had CTD: scleroderma (24), undifferentiated CTD (14), systemic lupus erythematosis (9), polymyositis/ dermatomyositis (9), rheumatoid arthritis (6), mixed CTD (3) and Sjogren's syndrome (2). The ILD diagnoses in patients with CTD were NSIP (36), organising pneumonia (OP) (16), LIP (6), usual interstitial pneumonia (UIP, 6) and mixed type (3). Seven patients $(3.9 \%)$ completed less than $85 \%$ of the questionnaire and were therefore excluded (two were visually impaired, two had language difficulty, for three the reason was not recorded).

Table 1 Patient demographics

\begin{tabular}{|c|c|}
\hline & All patients \\
\hline Number & 173 \\
\hline Mean age, years (SD) & $60(13)$ \\
\hline Women, \% & 60 \\
\hline \multicolumn{2}{|l|}{ Ethnicity, \% } \\
\hline Caucasian & 75 \\
\hline Afro-Caribbean & 10 \\
\hline South Asian* & 13 \\
\hline Other & 2 \\
\hline \multicolumn{2}{|l|}{ Smoking status, $\%$} \\
\hline Current & 6 \\
\hline Ex & 26 \\
\hline Never & 68 \\
\hline Mean time since diagnosis, years (SD) & $4.0(3.7)$ \\
\hline FVC, \% predicted (range) & $82(34-143)$ \\
\hline TLCO, \% predicted (range) & $49(12-92)$ \\
\hline \multicolumn{2}{|l|}{ Immunosuppressant medications, \% } \\
\hline None & 23 \\
\hline Prednisolone & 46 \\
\hline Prednisolone + other & 28 \\
\hline Other & 3 \\
\hline \multicolumn{2}{|l|}{ Comorbidity, \% } \\
\hline IHD & 5 \\
\hline Emphysema (by HRCT) & 11 \\
\hline $\begin{array}{l}\text { Immunosuppressant medications were tho } \\
\text { *South Asian patients originating from Inc } \\
\text { Emphysema (by HRCT), emphysema by } \\
\text { smoking history; FVC, forced vital capacit } \\
\text { factor of the lung for carbon monoxide as }\end{array}$ & $\begin{array}{l}\text { study. } \\
10 \text { pack-year } \\
\text { TLCO, transfer }\end{array}$ \\
\hline
\end{tabular}

\section{Phase 2: item reduction and Rasch analysis}

Thirty-two items were removed because of a significant floor effect or high inter-item correlation (figure 1). Exploratory factor analysis and a scree plot suggested a four-factor (domain) solution: psychological (10 items), breathlessness and activities (11 items), chest symptoms ( 9 items), and medication and finance (4 items). Five items were removed because they did not load sufficiently on any factor. Rasch analysis removed 19 poor fitting items (psychological ( 3 items), breathlessness and activities (7 items), chest symptoms (6 items), and medication and finance (3 items) (figure 1). There was no individual item misfit in remaining items and the item-trait interaction was nonsignificant, indicating good fit to the Rasch model. The final summary statistics for the 15 -item questionnaire indicated a good fit to the model and are reported in table 2 . The Person Separation Index for K-BILD total score was 0.94, suggesting it had good discriminant power to detect differing levels of health impairment. A person-item map indicated K-BILD items detected health status across a wide spectrum of health status severity (figure 2). There was no influence of age, gender, ethnicity, immunosuppressant medication, CTD or ILD diagnosis on the response to items (DIF). A confirmatory factor analysis confirmed items remained in their domains. All items within each domain related well to the health theme of its domain (face validity). The fourth factor contained only one item following Rasch analysis. This item was retained in the questionnaire since it contributed to the overall health status score and its fit to the Rasch model (question 15, see appendix). The fourth factor was no longer considered a domain since it consisted of just a single item. The response scales for eight items were disordered and corrected to ensure a progressive scale.

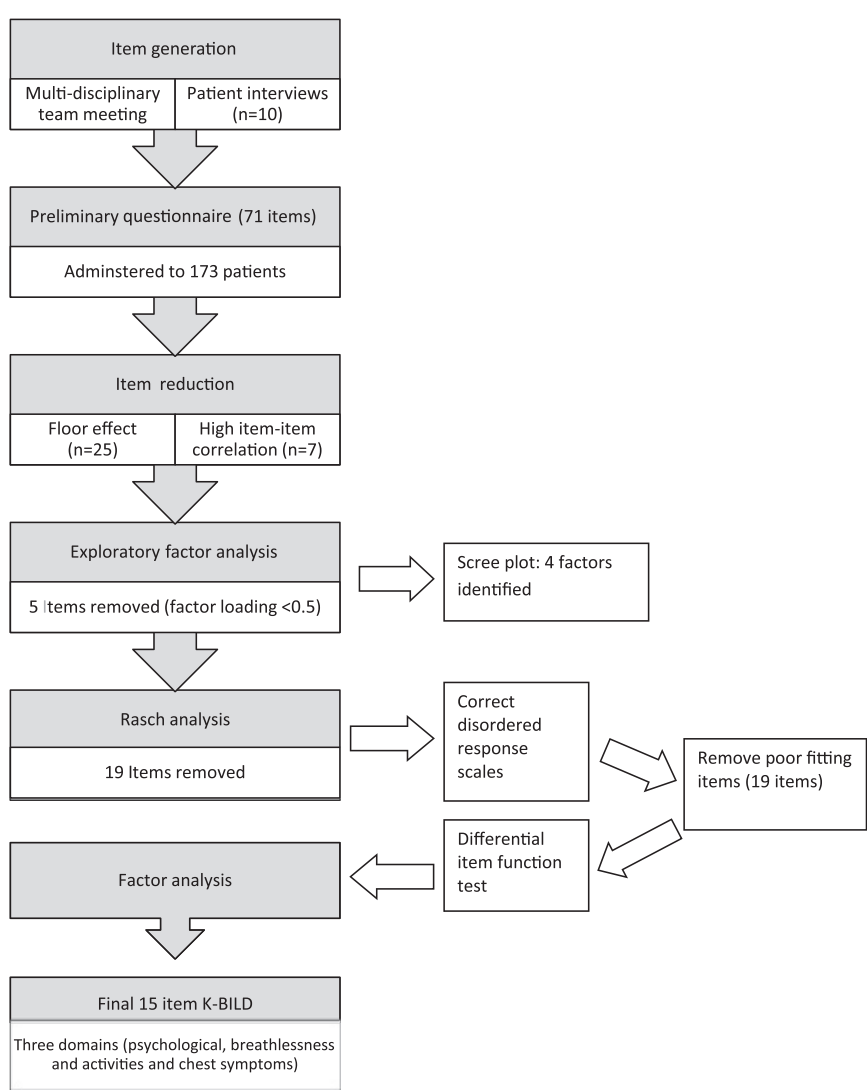

Figure 1 Summary of the development of the King's Brief Interstitial Lung Disease (K-BILD) questionnaire. 
Table 2 Summary of Rasch analysis

\begin{tabular}{|c|c|c|c|c|}
\hline \multirow[b]{2}{*}{ Rasch statistics } & \multicolumn{4}{|l|}{ K-BILD domains } \\
\hline & Psychological & Breathlessness and activity & Chest symptoms & Total score \\
\hline Number of items & 7 & 4 & 3 & 15 \\
\hline$\chi^{2}$ fit statistic & 18.2 & 8.2 & 7.9 & 41.0 \\
\hline Degrees of freedom & 14 & 8 & 6 & 30 \\
\hline p Value & 0.20 & 0.42 & 0.25 & 0.09 \\
\hline Mean item fit residual (SD) & $-0.1(0.9)$ & $-0.01(0.9)$ & $0.4(1.2)$ & $0.2(1.1)$ \\
\hline Mean person fit residual (SD) & $-0.3(1.1)$ & $-0.5(1.1)$ & $-0.5(1.3)$ & $-0.2(1.2)$ \\
\hline PSI & 0.91 & 0.93 & 0.70 & 0.94 \\
\hline Cronbach's $\alpha$ coefficient & 0.90 & 0.90 & 0.72 & 0.94 \\
\hline
\end{tabular}

The final K-BILD questionnaire consisted of 15 items and three domains: breathlessness and activities (questions 1, 4, 11, 13), psychological (questions 3, 5, 6, 8, 10, 12, 14) and chest symptoms (questions $2,7,9$ ); see appendix. A total of $96 \%$ of patients reported no difficulty in completing the K-BILD questionnaire.

\section{Phase 2: validation}

The mean (SD) K-BILD total scores in all patients with ILD and IPF were 59 (25) and 52 (26) respectively. The K-BILD total score correlated strongly with SGRQ, moderately with lung function and SF36 physical component and weakly with SF36 mental component (table 3). The K-BILD breathlessness and activity domain correlated best with lung function, SGRQ impact domain and SF36 physical component (table 3). The construct and concurrent validity, internal reliability and repeatability of the K-BILD questionnaire in patients with IPF were comparable to other ILDs (table 4). Health status was significantly worse in patients prescribed supplemental oxygen therapy than in patients not on supplemental oxygen $(n=25)$; mean (SEM) KBILD total score 38 (4) versus 63 (2); mean difference 25; 95\% CI of difference 15 to $35 ; \mathrm{p}<0.01$. Patients taking oxygen therapy had more severe disease; mean (SD) vital capacity 66\% (19\%) versus $81 \%(24 \%) ; p<0.01$. There was no significant difference in mean (SEM) K-BILD total score between patients with CTD$\operatorname{NSIP}(n=36)$ and idiopathic NSIP $(n=20): 62$ (4) versus 62 (5); mean difference 0.3 ; $95 \%$ CI of difference -13 to $12 ; \mathrm{p}=0.96$. The presence of emphysema on the CT scan did not impact significantly on K-BILD total score in patients with ILD (mean (SEM) 53 (7) vs 60 (2); $p=0.25$ ) or patients with IPF (mean
(SEM) 50 (10) vs 54 (4); p=0.78). FVC ( $<<0.03)$, TLCO $(p<0.02)$ and oxygen use $(p<0.02)$ were independent predictors of health status, accounting for $25 \%$ of the variance in health status. Immunosuppressant medication was not an independent predictor of health status.

\section{Phase 3: repeatability}

Repeatability was investigated in 44 patients (IPF (13 patients), CTD-ILD (16), idiopathic NSIP (5), HP (5), OP (3), LIP (2)). Intraclass correlation coefficients suggested good repeatability: K-BILD psychological 0.91, breathlessness and activity 0.96, chest symptoms 0.86 , and total 0.94 . The K-BILD intraclass correlation coefficients for patients with IPF were 0.83-0.98. A Bland-Altman plot of K-BILD total score repeatability is shown in figure 3 .

\section{DISCUSSION}

K-BILD is a health status questionnaire developed and validated specifically for patients with ILD. It is the first health status questionnaire developed for use in patients with ILDs other than IPF. The K-BILD questionnaire is brief, containing just 15 items that measure health status in three domains. It is simple to administer and most patients found it easy to complete.

The K-BILD items were generated following detailed patient interviews exploring the impact of their lung condition on their health. The preliminary K-BILD questionnaire was validated by a systematic statistical approach with factor and Rasch analysis, commonly used in the development of health status tools. Redundant items were removed if possible while retaining
Figure 2 Person-item threshold distribution for the King's Brief Interstitial Lung Disease questionnaire. The $x$-axis represents severity of health status in logits (log transformed units). The item threshold frequency represents the number of item responses that measure health status at each level of impairment. This figure demonstrates a good spread of item thresholds across the spectrum of health status.

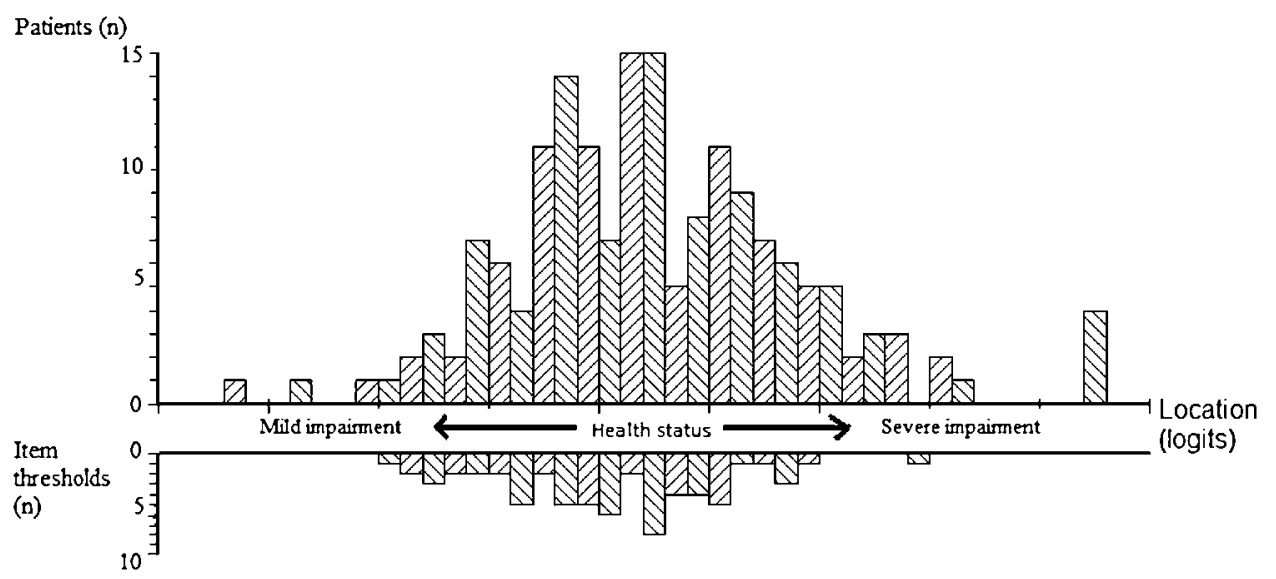


Table 3 The relationship between the King's Brief Interstitial Lung Disease (K-BILD) questionnaire and lung function and other health status measures

\begin{tabular}{|c|c|c|c|c|c|c|c|c|c|}
\hline \multirow[b]{2}{*}{ K-BILD } & \multicolumn{3}{|c|}{ Lung function } & \multicolumn{4}{|l|}{ SGRO } & \multicolumn{2}{|l|}{ SF36 } \\
\hline & $\mathrm{FEV}_{1} \%$ & FVC\% & $\mathrm{TLCO} \%$ & Sym & Act & Imp & Total & PCS & MCS \\
\hline Psychological & 0.46 & 0.38 & 0.45 & -0.60 & -0.67 & -0.80 & -0.79 & 0.57 & 0.38 \\
\hline Breathlessness and activity & 0.55 & 0.51 & 0.52 & -0.59 & -0.84 & -0.80 & -0.86 & 0.70 & 0.33 \\
\hline Chest symptoms & 0.48 & 0.45 & 0.42 & -0.65 & -0.64 & -0.79 & -0.78 & 0.61 & 0.36 \\
\hline Total & 0.53 & 0.47 & 0.50 & -0.67 & -0.79 & -0.87 & -0.89 & 0.68 & 0.40 \\
\hline
\end{tabular}

Data shown are Pearson's correlation coefficients; all $p<0.01$.

FEV $_{1}$, forced expiratory volume in 1 s; FVC, forced vital capacity; SF36, Short Form 36; SF36 MCS, SF36 Mental Component Summary; SF-36 PCS, SF36 Physical Component Summary; SGR0, St George's Respiratory Questionnaire; SGRO Act, SGRO - Activity; SGRO Imp, SGRO - Impact; SGRO Sym, SGRO - Symptoms; TLCO, diffusing capacity of the lung for carbon monoxide as $\%$ predicted.

essential questionnaire measurement characteristics. Factor analysis identified distinct health domains. Rasch analysis confirmed the K-BILD domains had good interval scaling of items and were unidimensional. Unidimensional scales are an important attribute of health status questionnaires because they confirm the instrument measures the intended construct and allows health status to be quantified as a single number to facilitate clinical utility and interpretation. The Rasch analysis also confirmed that the health domains could be combined to determine an overall unidimensional health status score. The correlation with lung function was moderate, analogous to that seen in chronic obstructive pulmonary disease (COPD), suggesting health status questionnaires assess a unique aspect of disease severity not captured by objective measures. ${ }^{16} 17$ The concurrent validity of the K-BILD questionnaire compared with the SGRQ was good, suggesting the removal of redundant items did not compromise K-BILD's ability to measure health status. K-BILD correlated more strongly with the SGRQ than the SF36. The weak association with the SF36, in particular with the mental health component summary score, raised the possibility that the SF36 did not fully identify the health issues specific for ILD since it was a generic instrument. The K-BILD questionnaire detected differences in health status among patients with advanced ILD prescribed home oxygen therapy compared with those not requiring oxygen therapy, which was in contrast to a previous study using generic health status tools that did not report a difference. ${ }^{18}$ This suggests that disease-specific tools such as the K-BILD questionnaire are better than generic tools at identifying the health issues important to patients with ILD.

We chose to develop an instrument that could assess health status in a wide range of ILDs while maintaining its validity for

Table 4 Concurrent validity, internal reliability and repeatability of the King's Brief Interstitial Lung Disease (K-BILD) questionnaire in idiopathic pulmonary fibrosis (IPF); comparison with other interstitial lung diseases (ILDs)

\begin{tabular}{|c|c|c|}
\hline & \multicolumn{2}{|c|}{ K-BILD Total score } \\
\hline & IPF & ILD, non-IPF \\
\hline SGRO Total & -0.9 & -0.9 \\
\hline SGRO Sym & -0.7 & -0.7 \\
\hline SGRO Act & -0.8 & -0.8 \\
\hline SGRO Imp & -0.9 & -0.9 \\
\hline FVC \% & 0.6 & 0.4 \\
\hline TLCO \% & $0.4^{*}$ & 0.5 \\
\hline $\begin{array}{l}\text { Internal reliability (Cronbach's } \\
\alpha \text { coefficient) }\end{array}$ & 0.9 & 0.9 \\
\hline $\begin{array}{l}\text { Repeatability (intraclass correlation } \\
\text { coefficient) }\end{array}$ & 0.9 & 0.9 \\
\hline
\end{tabular}

use in one of the commonest conditions, IPF. The K-BILD questionnaire was validated in a large group of patients with a wide range of ILDs, which increases our confidence that it is suitable for patients with most types of ILD. The DIF analysis did not identify significant differences in patient responses to items across categories of ILD. It is possible that health-related issues important to patients varied between ILDs and the construct of the questionnaire may have differed in rapidly progressive ILDs such as IPF. The K-BILD construct validity, internal reliability, repeatability and relationship with other measures was comparable in IPF to other ILDs, despite the likely differences in the severity of health status and the rate of progression of disease between groups. This suggests that the KBILD health status construct is similar across categories of ILD. It was not possible to validate K-BILD exclusively in single category ILDs other than IPF due to the large number of patients necessary for such studies and the low incidence of many conditions. Further studies need to investigate the validity of KBILD in specific ILDs. Most ILDs present with breathlessness but with varying degrees of severity. The person-item threshold distribution of K-BILD was large, confirming it assessed healthrelated quality of life (QOL) across a wide spectrum of health status. The high Person Separation Index for the K-BILD was consistent with the ability to discriminate differing levels of health status. Preliminary data reporting the responsiveness of the K-BILD questionnaire show promise but need confirming in larger longitudinal studies. ${ }^{19}$

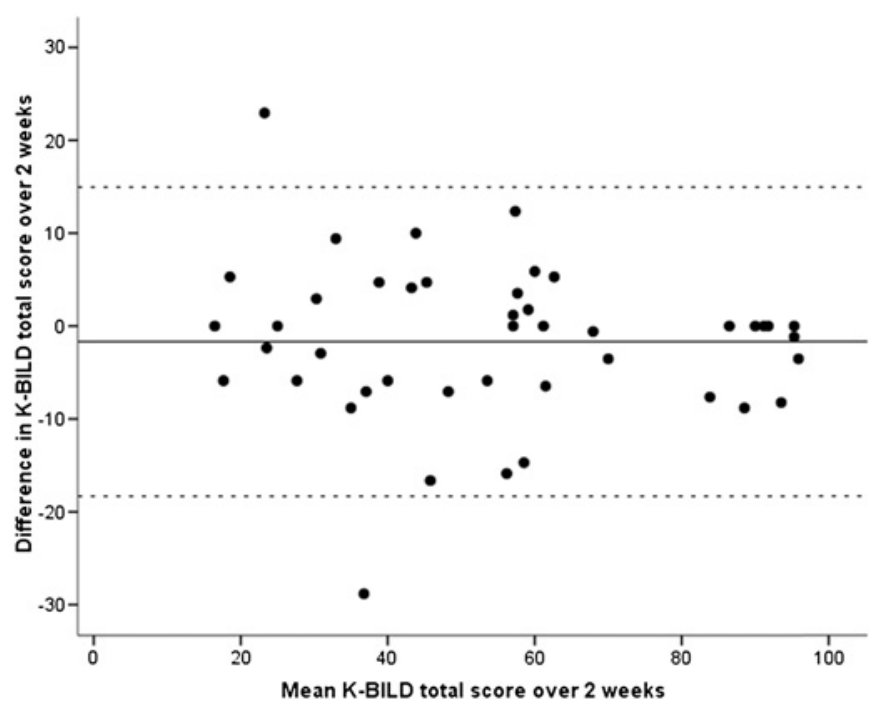

Figure 3 Bland Altman plot of repeatability of the King's Brief Interstitial Lung Disease (K-BILD) questionnaire repeatability. The solid line represents mean difference and dashed lines represent $95 \%$ limits of agreement. 
There are two other recently developed IPF specific health status questionnaires, the St George's Respiratory Questionnaire IPF (SGRO-I) and the 'A Tool to Assess QOL in IPF' (ATAQ-IPF). ${ }^{10}{ }^{16}$ The SGRQ-I, a 34-item questionnaire, was refined from the original 50-item SGRQ developed for chronic respiratory diseases such as COPD and then validated in patients with IPF. The ATAQ-IPF, a 74-item questionnaire, was developed and validated specifically for patients with IPF. SGRO-I, ATAO-IPF and K-BILD were all developed using Rasch analysis and demonstrated unidimensional and progressive response scales and reliable domain structures. K-BILD was validated in a large group of patients with IPF and its validity was comparable to that of SGRQ-I and ATAQ-IPF. The relationship of K-BILD with vital capacity in IPF $(r=0.60)$ was greater than that of SGRQ-I $(r=-0.33)$ and ATAQ-IPF $(r=-0.29)$. The relationship with gas transfer factor of the lungs was similar with all tools. A key difference between KBILD and ATAQ-IPF and SGRQ-I is that K-BILD is considerably shorter, consisting of just 15 items. This is likely to be important in research studies when patients are often subjected to multiple time-consuming assessments. A wide range of ILDs could be assessed with the K-BILD questionnaire and for most ILDs, it is the first specific tool developed to assess health status. K-BILD could be used at the time of presentation when the classification of the ILD is often unclear and to compare ILDs. In contrast, SGRQ-I and ATAQ-IPF were not developed for ILDs other than IPF and their validity in this setting is not known. The strong relationship between SGRO and K-BILD suggests that SGRO may also perform well in ILD. A key advantage of the K-BILD is that it is considerably shorter and has stronger concurrent validity with vital capacity in IPF. Further studies need to investigate whether K-BILD is a more responsive tool than SGRO in ILD.

There are limitations with health status questionnaire development methodology. It is possible that some items eliminated during the development process, such as cough, medications, sleep and sexual health, may have contributed significantly to health status in some patients. Our aim was to develop a questionnaire that could quantify health status with the least number of items, while retaining validity, so that it was practical for clinical use. Health status questionnaires are not a substitute for identifying health-related issues obtained from a detailed history. We eliminated items that were infrequent, redundant, contributed weakly to health status assessment or did not conform to optimal scaling properties. It is possible that health issues relevant to ILD that were not included in the final version of the K-BILD questionnaire were assessed indirectly by their impact on related health domains. For example, cough is known to be associated with low mood and interferes with daily tasks. ${ }^{5}$ Cough is troublesome in a subgroup of patients with ILD and it is perhaps more appropriate to assess it with a validated health status tool such as the Leicester Cough Questionnaire or the Cough-specific QOL Questionnaire. ${ }^{5} 20$

It is possible that systemic and musculoskeletal symptoms of CTD could have influenced health status. Such an effect is likely to be minimal since all K-BILD items specifically related to the impact of lung disease. Furthermore, the presence of CTD did not impact health status within the most common category of ILD, NSIP, and there was no DIF. It is possible that the coexistence of emphysema may have affected health status since an item related to wheeze was retained in the final version of the K-BILD. This is similar to the SGRQ-I. ${ }^{10}$ The prevalence of emphysema on highresolution CT scan was relatively low and there was no significant difference in the health status of patients with ILD with emphysema compared with those without, suggesting the impact of emphysema on health status was likely to be small. It is also possible that patients may have reported other symptoms as wheeze. This is a limitation of self-administered questionnaires, which was minimised as much as possible by using a lay description of wheeze. The internal reliability assessed with Cronbach's $\alpha$ coefficient for chest symptom domain $(\alpha 0.72)$, although satisfactory for group analysis, was borderline for individual patient assessment and therefore should be interpreted with caution in this setting. ${ }^{11}$ It is possible that medications, particularly corticosteroids, may have impacted health status greater than the ILD in some patients. This is unlikely because the K-BILD items specifically assessed the impact of lung disease on health status and there was no DIF with patients taking medications. Furthermore, multivariate analysis did not identify a relationship between medication and health status. Further studies, preferably with long-term follow-up, are needed to assess the temporal relationship between medications and health status. A further limitation is that we did not assess functional status of patients; future studies should include objective measures such as 6 min walk test. Our data were consistent with the K-BILD being highly repeatable over 2 weeks, but this needs re-evaluating over a longer duration.

The K-BILD questionnaire has a number of potential applications. It is a quick and valid tool to identify health status issues important to patients in the clinic. Furthermore, it could be used to help formulate shared care plans between the patient and physician. Our study suggests the K-BILD questionnaire is a valid single-point measure that could be used in cross-sectional studies. In summary, it is brief, easy to administer and well validated. It represents an advance in the assessment of ILD.

Reprints of K-BILD: the King's Brief Interstitial Lung Disease questionnaire 2011 is protected by copyright; King's College Hospital NHS Foundation Trust. Reprints of the K-BILD are available from the corresponding author.

Acknowledgements We would like to thank the staff in the clinics and lung function units at King's College Hospital and Royal Brompton Hospital for their assistance in characterisation of patients. We would also like to thank Dr Sabrina Bajwah for her participation in methodology discussions, Dr Katherine Irving and $\mathrm{Dr}$ Greg Keir for their assistance in characterising patients and Professors Mike Morgan and Sally Singh for reviewing the K-BILD questionnaire.

Contributors Conception and obtained funding: $\mathrm{SB}, \mathrm{IH}$; study design: all; recruitment: $A P, K B, S D, T M, E R, A W, S B$; analysis and interpretation: AP, RS, AW, IH, SB; data collection: $A P, K B$; wrote the manuscript: $A P, S B$; revised the manuscript: IH, RS, AW, $K B, P G, S S, S D, T M, E R$

Funding King's College Hospital Charity. AW, TM and ER are supported by the NIHR Respiratory Disease Biomedical Research Unit at the Royal Brompton and Harefield NHS Foundation Trust and Imperial College London.

Competing interests None of the authors has a financial relationship with a commercial entity that has an interest in the subject of this manuscript.

Ethics approval The ethics approval was provided by London-Surrey Borders Research Ethics Committee.

Provenance and peer review Not commissioned; externally peer reviewed.

Data sharing statement All data are published in this article.

\section{REFERENCES}

1. Coultas DB, Zumwalt RE, Black WC, et al. The epidemiology of interstitial lung diseases. Am J Respir Crit Care Med 1994;150:967-72.

2. Swigris JJ, Stewart AL, Gould MK, et al. Patients' perspectives on how idiopathic pulmonary fibrosis affects the quality of their lives. Health Qual Life Outcomes 2005; 3:61

3. American Thoracic Society; European Respiratory Society. American Thoracic Society/European Respiratory Society International Multidisciplinary Consensus Classification of the Idiopathic Interstitial Pneumonias. This joint statement of the American Thoracic Society (ATS), and the European Respiratory Society (ERS) was 
adopted by the ATS board of directors, June 2001 and by the ERS Executive Committee, June 2001. Am J Respir Crit Care Med 2002;165 277-304.

4. Bradley B, Branley HM, Egan JJ, et al; British Thoracic Society Interstitial Lung Disease Guideline Group, British Thoracic Society Standards of Care Committee; Thoracic Society of Australia; New Zealand Thoracic Society; Irish Thoracic Society. Interstitial lung disease guideline: the British Thoracic Society in collaboration with the Thoracic Society of Australia and New Zealand and the Irish Thoracic Society. Thorax 2008;63(Suppl 5):v1-58.

5. Birring SS, Prudon B, Carr AJ, et al. Development of a symptom specific health status measure for patients with chronic cough: Leicester Cough Questionnaire (LCO). Thorax 2003;58:339-43.

6. Nunally JC, Bernstein IH. Psychometric Theory. New York: McGraw-Hill, 1994

7. Yorke J, Moosavi SH, Shuldham C, et al. Quantification of dyspnoea using descriptors: development and initial testing of the Dyspnoea-12. Thorax 2010;65:21-6

8. Rasch G. Probabilistic Models for Some Intelligence and Attainment Tests. Chicago, IL: University of Chicago Press, 1960.

9. Meguro M, Barley EA, Spencer S, et al. Development and validation of an improved, COPD-specific version of the St George Respiratory Questionnaire. Chest 2007; 132:456-63

10. Yorke J, Jones PW, Swigris JJ. Development and validity testing of an IPFspecific version of the St George's Respiratory Questionnaire. Thorax 2010;65:921-6.
11. Tennant A, Conaghan PG. The Rasch measurement model in rheumatology: what is it and why use it? When should it be applied, and what should one look for in a Rasch paper? Arthritis Rheum 2007;57:1358-62.

12. American Thoracic Society. Single-breath carbon monoxide diffusing capacity (transfer factor). Recommendations for a standard technique - 1995 update. Am J Respir Crit Care Med 1995:152:2185-98.

13. American Thoracic Society. Standardization of spirometry, 1994 update. Am J Respir Crit Care Med 1995;152:1107-36

14. Jones PW, Quirk FH, Baveystock CM, et al. A self-complete measure of health status for chronic airflow limitation. The St. George's Respiratory Questionnaire. Am Rev Respir Dis 1992;145:1321-7.

15. Brazier JE, Harper R, Jones NM, et al. Validating the SF-36 health survey questionnaire: new outcome measure for primary care. BMJ 1992;305:160-4.

16. Swigris JJ, Wilson SR, Green KE, et al. Development of the ATAQ-IPF: a tool to assess quality of life in IPF. Health Qual Life Outcomes 2010;8:77.

17. Jones PW. Health status measurement in chronic obstructive pulmonary disease. Thorax 2001:56:880-7.

18. De Vries J, Kessels BL, Drent M. Quality of life of idiopathic pulmonary fibrosis patients. Eur Respir J 2001;17:954-61.

19. Patel AS, Siegert RJ, Bajwah S, et al. The King's Brief Interstitial Lung Disease questionnaire: responsiveness and minimal important difference (abstract). Am J Respir Crit Care Med 2012;185: Abstract number 27605.

20. French CT, Irwin RS, Fletcher KE, et al. Evaluation of a cough-specific quality-of-life questionnaire. Chest 2002;121:1123-31.

\section{APPENDIX}

\section{The King's Brief Interstitial Lung Disease Questionnaire (K-BILD) $\odot 2011$}

This questionnaire is designed to assess the impact of your lung disease on various aspects of your life. Please circle the response that best applies to you for each question

\begin{tabular}{|c|c|c|c|c|c|}
\hline \multicolumn{6}{|c|}{ climbing stairs or walking up an incline or hill. } \\
\hline 1. Every time & 2. Most times & 3. Several Times & 5. Occasionally & 6. Rarely & 7. Never \\
\hline \multicolumn{6}{|c|}{ 2. In the last 2 weeks, because of my lung condition, my chest has felt tight. } \\
\hline 1. All of the time & 2. Most of the time & 3. A good bit of the time 4 . Some of the time & 5. A little of the time & 6. Hardly any of the time & 7. None of the time \\
\hline \multicolumn{6}{|c|}{ 3. In the last 2 weeks have you worried about the seriousness of your lung complaint? } \\
\hline 1. All of the time & 2. Most of the time & 3. A good bit of the time 4 . Some of the time & 5. A little of the time & 6. Hardly any of the time & 7. None of the time \\
\hline \multicolumn{6}{|c|}{ 4. In the last 2 weeks have you avoided doing things that make you breathless? } \\
\hline 1. All of the time & 2. Most of the time & 3. A good bit of the time 4 . Some of the time & 5. A little of the time & 6. Hardly any of the time & 7. None of the time \\
\hline \multicolumn{6}{|c|}{ 5. In the last 2 weeks have you felt in control of your lung condition? } \\
\hline 1. None of the time & 2. Hardly any of the & 3. A little of the time & 5. A good bit of the time & 6. Most of the time & 7. All of the time \\
\hline \multicolumn{6}{|c|}{ 6. In the last 2 weeks, has your lung complaint made you feel fed up or down in the dumps? } \\
\hline 1. All of the time & 2. Most of the time & 3. A good bit of the time 4 . Some of the time & 5. A little of the time & 6. Hardly any of the time & 7. None of the time \\
\hline \multicolumn{6}{|c|}{ 7. In the last 2 weeks, I have felt the urge to breathe, also known as 'air hunger'. } \\
\hline 1. All of the time & 2. Most of the time & 3. A good bit of the time 4. Some of the time & 5. A little of the time & 6. Hardly any of the time & 7. None of the time \\
\hline \multicolumn{6}{|c|}{ 8. In the last 2 weeks, my lung condition has made me feel anxious. } \\
\hline 1. All of the time & 2. Most of the time & 3. A good bit of the time 4 . Some of the time & 5. A little of the time & 6. Hardly any of the time & 7. None of the time \\
\hline \multicolumn{6}{|c|}{ 9. In the last 2 weeks, how often have you experienced 'wheeze' or whistling sounds from your chest? } \\
\hline 1. All of the time & 2. Most of the time & 3. A good bit of the time 4 . Some of the time & 5. A little of the time & 6. Hardly any of the time & 7. None of the time \\
\hline \multicolumn{6}{|c|}{ 10. In the last 2 weeks, how much of the time have you felt your lung disease is getting worse? } \\
\hline 1. All of the time & 2. Most of the time & 3. A good bit of the time 4 . Some of the time & 5. A little of the time & 6. Hardly any of the time & 7. None of the time \\
\hline \multicolumn{6}{|c|}{ 11. In the last 2 weeks has your lung condition interfered with your job or other daily tasks? } \\
\hline 1. All of the time & 2. Most of the time & 3. A good bit of the time 4 . Some of the time & 5. A little of the time & 6. Hardly any of the time & 7. None of the time \\
\hline \multicolumn{6}{|c|}{ 12. In the last 2 weeks have you expected your lung complaint to get worse? } \\
\hline 1. All of the time & 2. Most of the time & 3. A good bit of the time 4 . Some of the time & 5. A little of the time & 6. Hardly any of the time & 7. None of the time \\
\hline \multicolumn{6}{|c|}{ 13. In the last 2 weeks, how much has your lung condition limited you carrying things, for example, groceries? } \\
\hline 1. All of the time & 2. Most of the time & 3. A good bit of the time 4 . Some of the time & 5. A little of the time & 6. Hardly any of the time & 7. None of the time \\
\hline \multicolumn{6}{|c|}{ 14. In the last 2 weeks, has your lung condition made you think more about the end of your life? } \\
\hline 1. All of the time & 2. Most of the time & 3. A good bit of the time 4 . Some of the time & 5. A little of the time & 6. Hardly any of the time & 7. None of the time \\
\hline \multicolumn{6}{|c|}{ 15. Are you financially worse off because of your lung condition? } \\
\hline 1. A significant amol & 2. A large amount & 3. A considerable amount 4.A reasonable amour & 5. A small amount & 6. Hardly at all & 7. Not at all \\
\hline
\end{tabular}

